
las Ciencias

ISSN: 1697-011X

revista.eureka@uca.es

Universidad de Cádiz

España

\title{
Descripción del conocimiento pedagógico del contenido de la argumentación en docentes que enseñan ciencias naturales en educación pública en Chile
}

Larrain, Antonia; Gómez, Marisol; Calderón, Maribel; Fortes, Gabriel; Ramírez, Francisca; Guzmán, Valentina; Cofré, Hernán

Descripción del conocimiento pedagógico del contenido de la argumentación en docentes que enseñan ciencias naturales en educación pública en Chile

Revista Eureka sobre Enseñanza y Divulgación de las Ciencias, vol. 19, núm. 1, 2022

Universidad de Cádiz, España

Disponible en: https://www.redalyc.org/articulo.oa?id=92068491018

DOI: https://doi.org/10.25267/Rev_Eureka_ensen_divulg_cienc.2022.v19.i1.1602 
Antonia Larrain, et al. Descripción del conocimiento pedagógico del Contenido de la argumentación ...

Formación del profesorado de ciencias

\section{Descripción del conocimiento pedagógico del contenido de la argumentación en docentes que enseñan ciencias naturales en educación pública en Chile}

Description of Chile primary science teachers' pedagogical content knowledge of argumentation

Antonia Larrain

Facultad de Psicología, Universidad Alberto Hurtado.

Santiago, Chile

alarrain@uahurtado.cl

iD https://orcid.org/0000-0002-1968-4516

Marisol Gómez

Facultad de Psicologia, Universidad Alberto Hurtado.

Santiago, Chile

marisolpgm1@gmail.com

iD https://orcid.org/0000-0002-6682-4323

Maribel Calderón

Centro de Investigación Socioeducativa, Facultad de

Educación, Universidad Católica Silva Henríquez, Chile

mcalderon@ucsh.cl

(iD https://orcid.org/0000-0002-8379-1463

Gabriel Fortes

Facultad de Psicología, Universidad Alberto Hurtado.

Santiago, Chile

gfortes@uahurtado.cl

(iD) https://orcid.org/0000-0003-4997-0019

Francisca Ramirez

Facultad de Psicologia, Universidad Alberto Hurtado.

Santiago, Chile

mfranciscaramirez@gmail.com

iD https://orcid.org/0000-0002-4578-3067

Valentina Guzmán

Facultad de Psicología, Universidad Alberto Hurtado.

Santiago, Chile

vaguzman@uahurtado.cl

iD https://orcid.org/0000-0001-6164-0843

Hernán Cofré

Instituto de Biología, Facultad de Ciencias, Pontificia

Universidad Católica de Valparaíso, Valparaiso, Chile

hernan.cofre@pucv.cl

iD https://orcid.org/0000-0003-2789-334X
DOI: https://doi.org/10.25267/

Rev_Eureka_ensen_divulg_cienc.2022.v19.i1.1602

Redalyc: https://www.redalyc.org/articulo.oa? $\mathrm{id}=92068491018$

Recepción: 14 Junio 2021

Revisado: 30 Agosto 2021

Aprobación: 31 Octubre 2021 


\section{Resumen:}

La enseñanza de las ciencias ha sido interpelada para formar para la ciudadanía pues esta demanda competencias propias de la ciencia como la capacidad de articular teoría y evidencia, y la argumentación y evaluación crítica de distintos puntos de vista. Promover la argumentación en la enseñanza de las ciencias es clave pero no es sencillo y requiere por parte de docentes lo que se ha llamado conocimiento pedagógico del contenido (CPC) de la argumentación. A nivel internacional se ha reportado bajo CPC de la argumentación en docentes de ciencias. Sin embargo, poco se sabe del CPC de la argumentación en países de habla hispana. El presente artículo reporta un estudio cuyo objetivo fue describir el CPC de argumentación en docentes que enseñan ciencias en educación primaria en Chile. Participaron 10 docentes de establecimientos de Santiago y Coquimbo. Para indagar las diferentes dimensiones del CPC (declarativo y procedimental) se realizaron grabaciones de aulas y entrevistas a docentes. Los resultados muestran que ningún docente despliega un CPC de la argumentación consistentemente alto en todas sus dimensiones. Más aún, el conocimiento procedimental no está relacionado necesariamente con alto conocimiento declarativo. Los resultados sugieren una progresión de aprendizaje de este tipo de conocimiento variable. Se reflexiona acerca del impacto de los resultados para la práctica y teoría educativa.

Palabras Clave: Conocimiento pedagógico del contenido de la argumentación, enseñanza de las ciencias, ciencias para la ciudadanía, aprendizaje de las ciencias.

\section{Abstract:}

Science teaching has been called to contribute to citizenship insofar as it requires scientific competencies such as articulating theory and evidence, and the critical evaluation of different points of view. To promote argumentation in science teaching is key but not simple and requires teachers' pedagogical content knowledge (PCK) of argumentation. International literature has reported low PCK of argumentation in science teachers, but we scarcely know about this in Spanish speaking countries. This paper reports a study aimed at describing the PCK of argumentation on 10 primary science teachers in Chile. Lessons were video recorded, and interviews were conducted. Results show low PCK of argumentation overall, and declarative PCK does not necessarily relates with procedimental PCK. Moreover, different configurations of the dimensions of PCK of argumentation were observed, suggesting that there is not one possible learning progression, but different ones. Educational and scientific implications are discussed.

KEYWORDS: Pedagogical content knowledge of argumentation, science teaching, science for citizenship, science learning.

\section{INTRODUCCIÓN}

Una ciudadanía activa, que se involucre con la solución de problemas comunes de manera crítica pero sostenible requiere, lo que se ha llamado, alfabetización científica, que involucra tanto el dominio de conocimiento científico y la comprensión de la naturaleza de la ciencia (Davies 2004), como la práctica de argumentación (Tippett 2009). Esto interpela a la ciencia y a su enseñanza de manera directa y urgente. No solo se trata de enseñar a construir y evaluar argumentos científicos, lo que es central, sino a participar en la articulación colectiva de nuestras diferencias. Kolstø (2000) plantea la necesidad de proveer experiencias de ejercicio del debate y la discusión de controversias como un aspecto organizador de la enseñanza de ciencias para la ciudadanía. Sabemos que estas experiencias no solo promueven la capacidad de argumentar científicamente (Hemberger, Kuhn, Matos y Shi 2017), sino también de comprender conceptos científicos (Asterhan y Schwarz 2016). La evidencia disponible sugiere, sin embargo, que la enseñanza de ciencias que predomina en países Latinoamericanos dista de ofrecer experiencias de argumentación e indagación (ver Cofré et al. 2010, González-Weil et al. 2012, Larrain, Howe y Freire 2014).

Argumentación es un término polisémico (Jiménez-Aleixandre y Erduran 2008) pero para efectos de este trabajo la entenderemos, basados en el trabajo de Asterhan y Schwarz (2016), tanto como el proceso como la habilidad involucrados en la práctica discursiva de lidiar con asuntos controversiales, que implica la elaboración y evaluación crítica de razones para sostener puntos de vista opuestos, y la articulación de teoría y evidencia. La concebimos como un proceso dialógico (Leitão 2000) en tanto involucra diferentes perspectivas en tensión y, de acuerdo a nuestro punto de vista, la argumentación incluye distintas dimensiones: una dimensión comunicativa, en tanto típicamente involucra la comunicación entre distintos hablantes; una dimensión epistémica, en tanto es un proceso que promueve la construcción de conocimiento; 
una dimensión dialéctica, pues involucra la existencia y emergencia de controversias que son críticamente examinadas; y una dimensión pedagógica, en la medida que puede emerger como parte de diseños deliberados para promover aprendizaje y desarrollo.

En la enseñanza de ciencias, tradicionalmente se ha enfatizado la importancia de que estudiantes aprendan a construir argumentos científicos, en donde conclusiones sean apropiadamente sostenidas por evidencia a través de un proceso de razonamiento (McNeill y Krajcik 2008). Esto ha sido concebido como énfasis en la dimensión estructural de la argumentación. Sin embargo, autores como Osborne (2010) han insistido en la importancia de que estudiantes aprendan no solo a construir argumentos científicos de calidad, sino a participar de la práctica crítica de discusión de puntos de vista opuestos, a través del intercambio de argumentos y contra-argumentos en el aula, lo que ha sido entendido como enfatizando su aspecto dialógico. Ambos aspectos se reconocen como parte central de la ciencia en tanto práctica y de la ciudadanía, y, por tanto, objetivos educacionales claves.

\section{Conocimiento pedagógico del contenido de la argumentación}

Shulman (1986) define el conocimiento pedagógico del contenido (CPC) como un componente único dentro de los distintos conocimientos profesionales que requieren los profesores para realizar una enseñanza efectiva. Posteriormente, Magnusson, Krajcik y Borko (1999), conceptualizaron el CPC como un conocimiento formado por cinco componentes: (a) orientaciones hacia la enseñanza, (b) conocimiento del currículo, (c) conocimiento de la evaluación, (d) conocimiento de la comprensión de los estudiantes y (d) conocimiento de las estrategias de enseñanza. Si bien el desarrollo histórico de este constructo ha incluido múltiples modelos (véase Vergara, Santibáñez y Cofré, 2021), el modelo de Magnusson et al., (1999), y sus modificaciones posteriores, como el modelo hexagonal propuesto por Park y Oliver (2008) se han utilizado profusamente en la literatura de acuerdo con algunas de las revisiones más citadas sobre CPC (Abell 2007; van Driel, Berry y Meirink 2014; Chan y Hume 2019). De hecho, Chan y Hume (2019) recientemente mostraron que, en los últimos 10 años $(2008$ - 2018) de los casi 100 artículos revisados, el 50\% han utilizado el modelo de Magnusson o modificaciones de él para investigar el CPC, superando a otros modelos de propuestos como el modelo de consenso (Gess-Newsome, 2015; Berry, Friedrichsen, y Loughran, 2015) y el modelo de consenso refinado (Carlson y Daehler 2019). De acuerdo con toda esta literatura, se ha reconocido que un CPC sofisticado incluye un conocimiento acabado de las preconcepciones y los desafíos que enfrentan los estudiantes al aprender un contenido y sobre las estrategias de enseñanza más efectivas utilizadas para promover el aprendizaje de los estudiantes (Loughran, Mulhall y Berry 2004, Vergara y Cofré 2014; AcevedoDíaz y García-Carmona, 2016; Ravanal y López 2016).

Por otra parte, otros autores también han querido distinguir entre lo que el/la profesor(a) hace en el aula y aquellos elementos que considera en la toma de decisiones antes de la enseñanza (Loughran et al., 2008). De esta forma, se define un CPC declarativo, relativo a la forma de resolver la serie de tareas en el proceso de preparación de la enseñanza y un CPC procedimental, que alude a las acciones que el profesor exhibe durante la enseñanza, al interior de una sala de clases (Schmelzing et al., 2013). Otros autores han descrito esta separación como el CPC on action (sobre la acción) y el CPC in action (en acción) (Park y Oliver 2008). Estas últimas autoras generaron una nueva versión del modelo de Magnusson la cual incluye: los cinco componentes originales en la periferia de un hexágono, los aspectos declarativos y procedimental, así como un sexto componente afectivo o de autoeficacia del profesor el cual sería específica del contexto (Park y Oliver 2008).

La argumentación como objetivo pedagógico es muy desafiante pues requiere de un diseño cuidadoso (Andriessen y Schwarz 2009). Por lo anterior, se ha descrito como requisito esencial para la enseñanza efectiva de la argumentación por parte de los profesores, el desarrollo de su CPC de argumentación (McNeill, Gozález-Howard, Katsh-Singer y Loper 2016, Sengul, Enderle y Schwartz 2020). El CPC de argumentación 
(McNeill et al. 2016) se concibe como el conocimiento y creencias que docentes tienen sobre el uso y la enseñanza de la argumentación en sus clases, incluyendo tanto aspectos declarativos (es decir el conocimiento teórico o el saber qué hay que enseñar), como procedimentales (es decir el conocimiento práctico o en la acción) (Vergara y Cofré 2014). Dentro de los primeros, se encontraría cierto manejo respecto a qué es la argumentación en aula, cuál es su importancia y relación con el aprendizaje, en qué consiste la estructura de un argumento científico, entre otros. Dentro de los aspectos procedimentales se incluiría conocimiento acerca de cómo diseñar experiencias de aprendizaje (Knight-Bardsley y McNeill 2016), cómo identificar interacciones productivas y cómo gestionar la argumentación en el aula (McNeill et al.2016, Sengul et al. 2020).

El CPC de la argumentación involucra un conocimiento tácito, difícil de evaluar y muy desafiante para docentes (Wang y Buck 2016). De hecho, se ha reportado bajo conocimiento de contenido de la argumentación en docentes de ciencias en formación inicial (Sadler, 2006) y de secundaria (Wang y Buck 2016) y baja percepción de conocimiento para el uso de la argumentación en aula (Sampson y Blanchard 2012). La evidencia empírica sugiere que docentes: creen que la argumentación no es un objetivo educacional para todos los estudiantes (McNeill et al. 2016); no creen que promueva aprendizaje (Sampson y Blanchard 2012); tienen dificultad en reconocer y evaluar interacciones dialógicas productivas en aula (Alozie, Moje y Krajcik,2010, McNeill y Knight 2013) y escasamente las promueven (Larrain et al. 2014). Además, se reporta que mucho de lo que docentes y estudiantes hacen en sus clases y entienden por argumentación, podría concebirse como pseudo-argumentación (McNeill et al. 2016), definida a partir de McNeill et al (2016) y Sengul et al. (2020) como: <<la orientación a las características básicas y superficiales de la argumentación en aula, desatendiendo a su función epistémica y al proceso de construcción de conocimiento que se lleva a cabo a través de esta >> (Fortes et al. 2021, p. 178).

\section{Medición de conocimiento pedagógico del contenido de la argumentación}

La medición del CPC es compleja dado que implica saberes procedimentales tácitos. Por esta razón, diferentes revisiones han propuesto que la mejor forma de medir y describir el CPC es la recolección de datos proveniente de diferentes fuentes (Baxter y Lederman 1999; Vergara y Cofré 2014; Chan y Hume 2019). Según la revisión de Chan y Hume (2019), las diferentes metodologías para capturar el $\mathrm{CPC}$ incluyen la triangulación de entrevistas estructuradas y semiestructuradas, entrevistas de recuerdo estimuladas, revisión de videos mediante rúbricas y la generación de mapas conceptuales. Según esta misma revisión, se concluye que una de las propuestas más utilizadas para medir el CPC son los instrumentos CoRes (Content Representation) y PaP-eRs (Pedagogical and Professional experience repertories) desarrollados por John Loughran y sus colegas de la Universidad de Monash (Loughran, Mulhall y Berry, 2004). Estos instrumentos han sido ampliamente utilizados para describir y documentar el CPC de profesores secundarios y primarios, especialmente en profesores de ciencia (e.g., Loughran, et al., 2004, 2008; Padilla et al., 2008; Bravo y Cofré 2016; Ravanal y López 2016; Walan, Nilsson y Ewen 2017; Rollnick, 2017). El principal resultado de estos esfuerzos ha sido el convencimiento de que la mejor forma de caracterizar el CPC es tanto a través de la observación del desempeño del profesor como de entrevistas donde el profesor explique por qué tomó las decisiones pedagógicas que se observan.

En relación al CPC de argumentación, investigadores como Wang y Buck (2016) y McNeill et al. (2016) han privilegiado la observación de clases y la realización de entrevistas, incluyendo preguntas acerca de la práctica pedagógica. McNeill et al. (2016) desarrollaron un proceso iterativo de desarrollo de material de evaluación que consiste en un conjunto de micro situaciones de enseñanza (viñetas), aludiendo a aspectos estructurales y dialógicos de argumentación científica, respecto de las que docentes deben escoger alternativas de respuesta. Los resultados del proceso dieron cuenta de que una alta competencia en CPC de argumentación involucraba: identificar la estructura y evaluar la calidad de los argumentos de estudiantes; 
identificar el uso de estrategias instruccionales para responder a las intervenciones argumentativas de los y las estudiantes; identificar objetivos interaccionales y evaluar la calidad de las interacciones dialógicas.

Knight-Bardsley y McNeill (2016) se focalizaron en evaluar CPC de argumentación a través de preguntas basadas en muestras de argumentos y discusiones de estudiantes, para indagar en la capacidad docente de identificar y evaluar acciones argumentativas de estudiantes y responder a estas. Esta modalidad podría inscribirse dentro de la investigación en visión profesional, entendida como la atención selectiva a elementos relevantes de la sala de clases y activar conocimientos disciplinares y pedagógicos pertinentes para interpretar y evaluar estos hechos (Van Es y Sherin 2008). Además, para indagar el diseño pedagógico de clases, observaron y analizaron clases, y entrevistaron a docentes después de cada clase para indagar en sus procesos de reflexión respecto a estas. Las autoras usaron una progresión de aprendizaje para analizar los datos que consistía en seis niveles: nivel $1=$ foco en transmisión de conocimientos; nivel $2=$ foco en experimentación e indagación; nivel 3 = foco en construcción de explicaciones científicas; nivel 4 = foco en rol de la evidencia en la construcción de argumentos; nivel 5 = foco en articulación entre evidencia y conclusión; nivel 6 = valor de la contra-argumentación y discusión de ideas contrarias.

Un estudio de García (2017) reporta las creencias respecto a la argumentación en 27 futuros docentes de biología en Argentina. Los resultados muestran que existe alta valoración de la argumentación en la ciencia y en sus propias estrategias de enseñanza. Las concepciones se centran más que en los aspectos dialógicos, en el carácter epistémico de la argumentación: la importancia de sostener conclusiones con evidencias. Un estudio de Garcia Romano, Condat, Occelli y Valerias (2016) con 25 docentes de ciencias muestra que estos sostienen una noción de argumentación que enfatiza su carácter explicativo ignorando su dimensión retórica; que los problemas socio-científicos son identificados como recursos pedagógicos para su enseñanza; y que docentes se centran en deficiencias de estudiantes para involucrarse en la argumentación. Identifican, además, dos polos: uno centrado en la argumentación en el discurso docente y sus necesidades y dificultades, el que estaría relacionado con un enfoque transmisivo de la enseñanza; y una centrada en estudiantes, relacionado con un enfoque más constructivista. El principal foco pedagógico de la argumentación en aula sería el dar a conocer las posiciones y posturas de estudiantes, con un escaso foco en los procesos epistémicos involucrados más allá de expresar puntos de vista. Martín-Gámez (2020) en un estudio con 65 docentes de educación primaria en formación inicial muestra que estos le dan importancia a la argumentación en la enseñanza de ciencias, pero con poca precisión para identificar procesos formativos específicos y estrategias que la promuevan. Ahora bien, la investigación en CPC de la argumentación en habla hispana es escasa, particularmente aquella que articula aspectos de conocimiento declarativos y procedimentales en una misma muestra. Archila (2014) reporta un estudio con 18 docentes de química en formación inicial muestra que estos si bien valoran la argumentación en aula, no se sienten preparado/as para promoverla. Para contribuir con esta brecha de conocimiento, el presente estudio tuvo como objetivo describir el CPC de argumentación en docentes que enseñan ciencias en Chile. La pregunta de investigación que guio el estudio fue: ¿cuál es el nivel de CPC de contenido de argumentación, tanto en sus aspectos procedimentales y declarativos, de docentes que enseñan ciencias en enseñanza primaria en educación pública en Chile?

\section{MÉTodo}

Para responder la pregunta de investigación, se desarrolló un estudio de corte transversal y de carácter descriptivo. El objetivo del estudio fue describir el nivel de CPC de argumentación en docentes que enseñan ciencias en enseñanza básica en educación pública en Chile. Entenderemos el CPC como un conocimiento profesional propio de la enseñanza de la disciplina, compuesto por diferentes elementos (por ejemplo, conocimiento de estrategias de enseñanza) los cuales se pueden expresar de forma declarativa o procedimental. Si bien la conceptualización del CPC de argumentación no se ha explicitado directamente a 
algún modelo concreto de CPC (Wangy Buck 2016; McNeill et al. 2016; Sengul et al., 2020), en este estudio se toman elementos presentes en los modelos de Magnusson et al (1999) y Park y Oliver (2008).

\section{Participantes}

Diez docentes participaron en el estudio, reclutadas a través de un muestreo por conveniencia de acuerdo a los siguientes criterios: trabajar en establecimientos públicos de las regiones Metropolitana y Coquimbo; tener y no tener formación previa en indagación científica; contar con contactos previos con establecimientos y/ o docentes. La formación en indagación científica se consideró una variable relevante toda vez que docentes de establecimientos públicos del país han participado de un proceso de desarrollo profesional docente a nivel nacional en el tema que aunque no focaliza en argumentación, sí entrega un marco general que puede facilitar el CPC de argumentación. Al momento del estudio (año 2018), los y las docentes enseñaban ciencias en cuarto año básico (estudiantes de 10-11 años) en establecimientos públicos pertenecientes a la Región de Metropolitana (6) y de Coquimbo. Se contactó a los y las participantes a través de directivos de establecimientos y de contactos directos con docentes. El promedio de edad fue de 46,8 años con 9,7 años en promedio de ejercicio docente (ver Tabla 1). Cuatro docentes habían participado de un programa de formación docente a cargo del Gobierno de Chile en indagación en ciencias.

TABLA 1

Características docentes participantes.

\begin{tabular}{|c|c|c|c|c|c|}
\hline Caso & Edad & Sexo & Formación inicial & $\begin{array}{c}\text { Años de ejercicio docente } \\
\text { (en ciencias naturales) }\end{array}$ & $\begin{array}{c}\text { Formación } \\
\text { indagación } \\
\text { científica }\end{array}$ \\
\hline 1 & 45 & H & $\begin{array}{c}\text { Pedagogía general básica } \\
\text { (PGB), mención } \\
\text { tecnología y matemáticas }\end{array}$ & $9(3)$ & sí \\
\hline 2 & 48 & H & PGB & $17(5)$ & sí \\
\hline 3 & 45 & M & $\begin{array}{c}\text { PGB, mención educación } \\
\text { física }\end{array}$ & $22(5)$ & - \\
\hline 4 & 67 & H & PGB & $43(43)$ & sí \\
\hline 5 & 52 & M & PGB & $4(2)$ & - \\
\hline 6 & 45 & M & PGB & $16(9)$ & - \\
\hline 7 & 38 & H & PGB & $12(4)$ & - \\
\hline 8 & 36 & M & $\begin{array}{c}\text { PGB, mención en } \\
\text { trastorno de aprendizaje }\end{array}$ & $10(8)$ & - \\
\hline 9 & 32 & M & PGB & $8(8)$ & $38(38)$ \\
\hline 10 & 60 & M & PGB & & \\
\hline
\end{tabular}

\section{Procedimiento de recogida y análisis de datos}

Una vez establecido el contacto con docentes se les invitó a participar del estudio siguiendo un proceso de consentimiento informado de acuerdo con la normativa de ética de investigación con personas vigente en el país y a la normativa establecida por la APA para la investigación científica. Luego, se procedió a coordinar la producción de datos. Estos se produjeron de dos maneras: grabaciones de clases y entrevistas semi-estructuradas. 
Primero, se coordinó con cada docente la filmación de dos clases, cuyo diseño y gestión estuvo a cargo completamente de los y las docentes. Estas grabaciones las realizó el equipo de investigación. Luego, se realizaron dos entrevistas semi-estructuradas por cada docente participante. Las entrevistas las realizaron personas parte del equipo de investigación especialmente entrenadas y supervisadas por una de las coautoras. Estas fueron videograbadas, transcritas y analizadas por el equipo de investigación.

\section{Instrumento}

\section{CPC de argumentación declarativo}

El componente declarativo del CPC de la argumentación se evaluó a través de la primera entrevista cuyo guion se desarrolló siguiendo las propuestas de Loughran et al. (2004, 2008), en sus Representaciones del Contenido ( $\mathrm{ReCo}$ ), las cuales han sido traducidas y utilizadas en contexto chileno (Vergara y Cofré 2014, Bravo y Cofré 2016 y McNeill et al. (2017)). Se indagó sobre las nociones de los y las docentes respecto a la argumentación y su potencial para el aprendizaje de ciencias, las condiciones que facilitan y dificultan la enseñanza a través de la argumentación, la disposición y conocimiento que los estudiantes despliegan durante actividades argumentativas y, por último, las estrategias que consideran apropiadas y efectivas para promover la argumentación.

Se realizó un proceso de codificación abierta por dos coautoras, que incluyó triangulación de datos en varias etapas. Una vez que se contó con las entrevistas codificadas, se desarrolló una rúbrica a partir del trabajo de Knight-Bardsley y McNeill (2016). Se realizó un proceso de validación intersubjetiva entre dos coautoras por medio de reflexiones conjuntas en reuniones semanales, además de presentación y retroalimentación del resto del equipo. En su versión final la rúbrica se compone de dos partes, las cuales se usan de manera secuencial. La primera parte permite juzgar cada una de las dimensiones de la entrevista según cuatro niveles (experto, en transición, inicial, y no experto). La segunda parte (adjunta en Tabla 2) permite realizar una descripción unificada (juicio global) del CPC de argumentación declarativo, según los cuatro niveles mencionados. 
TABLA 2

Rúbrica de CPC de argumentación declarativo - juicio global.

\begin{tabular}{|c|c|c|c|}
\hline Experto & En transición & Inicial & No experto \\
\hline $\begin{array}{c}\text { Refiere a la } \\
\text { argumentación como una } \\
\text { práctica epistémica, de } \\
\text { carácter dialéctico } \\
\text { (existencia y tramitación } \\
\text { de controversias) } \\
\text { identificando acciones } \\
\text { pedagógicas y } \\
\text { comunicativas relevantes } \\
\text { para su implementación } \\
\text { en el aula. Estas cuatro } \\
\text { dimensiones } \\
\text { (comunicativa, } \\
\text { epistémica, dialéctica y } \\
\text { pedagógica) se organizan } \\
\text { sistemáticamente entre sí. }\end{array}$ & $\begin{array}{c}\text { Refiere la dimensión } \\
\text { epistémica de la } \\
\text { argumentación en su } \\
\text { relación con el } \\
\text { pensamiento y/o como } \\
\text { proceso de construcción } \\
\text { de conocimiento. En } \\
\text { ocasiones se reconoce la } \\
\text { dimensión dialéctica de } \\
\text { la argumentación y/o } \\
\text { identifica acciones } \\
\text { pedagógicas relevantes } \\
\text { para su implementación } \\
\text { en el aula. Sin embargo, } \\
\text { estas dimensiones } \\
\text { (comunicativa, } \\
\text { epistémica, dialéctica y } \\
\text { pedagógica) no se } \\
\text { organizan de manera } \\
\text { sistemática entre sí. }\end{array}$ & $\begin{array}{l}\text { Refiere principalmente a la } \\
\text { dimensión comunicativa de } \\
\text { la argumentación como } \\
\text { centrada en el diálogo y/o } \\
\text { la experimentación } \\
\text { científica. No menciona } \\
\text { dimensión dialéctica ni su } \\
\text { relación con dimensión } \\
\text { epistémica. No identifica } \\
\text { con precisión acciones } \\
\text { pedagógicas claves para } \\
\text { implementación de uso } \\
\text { pedagógico de } \\
\text { argumentación en aula, sino } \\
\text { acciones genéricas de } \\
\text { colaboración y } \\
\text { comunicación. }\end{array}$ & $\begin{array}{c}\text { Refiere a la } \\
\text { argumentación en su } \\
\text { dimensión } \\
\text { comunicativa como } \\
\text { centrada en la } \\
\text { búsqueda exclusiva de } \\
\text { ideas correctas o de } \\
\text { transmisión de } \\
\text { conocimiento, } \\
\text { desconociendo su } \\
\text { dimensión propiamente } \\
\text { epistémica, en tanto } \\
\text { propceso de } \\
\text { pensamiento y } \\
\text { construcción de } \\
\text { conocimiento más allá } \\
\text { de la transmisión. }\end{array}$ \\
\hline
\end{tabular}

\section{CPC de argumentación procedimental}

Para evaluar el CPC en su versión procedimental, se utilizaron tres herramientas complementarias: 1) el análisis de interacciones de aula en clase completa, 2) la reconstrucción del diseño de clases (ambas a partir de las grabaciones de clases) y 3) una tarea de visión profesional que fue realizada en el contexto de la segunda entrevista.

\section{Argumentación en clase completa}

Para describir el uso pedagógico de la argumentación en aula se usó una pauta de análisis desarrollada por Larrain, Freire y Howe (2014) para identificar uso de enunciados argumentativos en clase completa. Dos co-autoras doble-codificaron las clases con la orientación de la autora principal. Para el establecimiento de confiabilidad inter-jueces, se doble codificaron un $26 \%$ del total de grabaciones de todo el proyecto. En la última ronda todos los códigos fueron considerados excelentes $(K$ de Cohen $>.810, p<.001)$. Para caracterizar el tipo de interacciones argumentativas, se realizó un análisis de los videos en base a una lista de chequeo para identificar la frecuencia con que estos enunciados argumentativos se configuraban en interacciones pseudo-argumentativas (McNeill et al. 2016) y argumentativas. Cada clase grabada fue observada y analizada por una coautora. Un porcentaje del material fue doble analizado por la autora principal para el desarrollo de intersubjetividad. A partir de ambos análisis (enunciados argumentativos y frecuencia de tipos de interacciones) cada clase fue juzgada en relación con el uso pedagógico de la argumentación (argumentación en clase completa) de acuerdo a una rúbrica según cuatro niveles, que se describen en la Tabla 3. 
TABLA 3

Rúbrica CPC de argumentación procedimental/uso pedagógico de argumentación en aula juicio global.

\begin{tabular}{|c|c|c|c|}
\hline Experto & En transición & Inicial & No experto \\
\hline $\begin{array}{l}\text { Se observan enunciados } \\
\text { argumentativos y contra- } \\
\text { argumentativos de parte de } \\
\text { estudiantes, además de } \\
\text { acciones docentes que } \\
\text { configuran interacciones en } \\
\text { las que los argumentos se } \\
\text { elaboran habiendo un foco } \\
\text { en el proceso epistémico. }\end{array}$ & $\begin{array}{c}\text { Se observan enunciados } \\
\text { argumentativos } y, \\
\text { eventualmente, contra- } \\
\text { argumentativos de parte de } \\
\text { estudiantes, y acciones } \\
\text { docentes, que configuran } \\
\text { interacciones pseudo- } \\
\text { argumentativas, habiendo un } \\
\text { foco superficial en la } \\
\text { característica de la interacción, } \\
\text { pero no en el proceso } \\
\text { epistémico. }\end{array}$ & $\begin{array}{c}\text { Se observan } \\
\text { enunciados de } \\
\text { estudiantes y } \\
\text { docentes tendientes a } \\
\text { la construcción de } \\
\text { argumentos, sin } \\
\text { contra-argumentación } \\
\text { de estudiantes. }\end{array}$ & $\begin{array}{l}\text { Solo se observan } \\
\text { enunciados } \\
\text { argumentativos en } \\
\text { forma de preguntas } \\
\text { justificativas } \\
\text { docentes. }\end{array}$ \\
\hline
\end{tabular}

\section{Diseño de clases}

Para describir el tipo de diseño de acuerdo a la potencialidad para promover argumentación en aula, se elaboró una pauta de análisis para describir dos dimensiones: el potencial de las actividades de clase completa y de trabajo en grupo. Siguiendo a Knight-Bardsley y McNeill (2016) se elaboró una rúbrica en base a la cual dos coautores juzgaron ambas dimensiones de cada clase en un proceso colaborativo e intersubjetivo. Luego la autora principal observó y aplicó la pauta independientemente, discutiendo desacuerdos, los que fueron mínimos y puntuales. La pauta incluye, además, una segunda parte en que ambas dimensiones se describen para levantar un solo juicio respecto al diseño de la clase (Tabla 4), de acuerdo con los cuatro niveles señalados.

TABLA 4

Rúbrica CPC de argumentación procedimental/ diseño de clases-juicio global.

\begin{tabular}{|c|c|c|c|}
\hline Experto & En transición & Inicial & No experto \\
\hline $\begin{array}{l}\text { Existe una propuesta de } \\
\text { actividad de clase que } \\
\text { involucra, tanto a nivel de } \\
\text { clase completa o plenario, } \\
\text { y trabajo en grupo, una } \\
\text { situación polémica o } \\
\text { controversial que deben } \\
\text { decidirse a través de } \\
\text { argumentación. }\end{array}$ & $\begin{array}{l}\text { Existe una propuesta de } \\
\text { actividad de clase completa } \\
\text { y/o plenario, y trabajo entre } \\
\text { pares, que involucran } \\
\text { perspectivas } \\
\text { complementarias que deben } \\
\text { justificarse, pero sin que } \\
\text { exista controversia } \\
\text { explícita. }\end{array}$ & $\begin{array}{c}\text { Existe una propuesta de } \\
\text { actividad de clase completa } \\
\text { y/o plenario que involucra } \\
\text { perspectivas } \\
\text { complementarias que deben } \\
\text { justificarse, pero sin que } \\
\text { exista controversia explícita. } \\
\text { Si hay trabajo en grupo, no } \\
\text { se orienta a la } \\
\text { argumentación } \\
\text { interpersonal. }\end{array}$ & $\begin{array}{l}\text { Clase completa, } \\
\text { plenario y/o } \\
\text { trabajo en grupo } \\
\text { no están } \\
\text { diseñados para } \\
\text { fundamentar } \\
\text { posturas ni } \\
\text { discutir } \\
\text { controversias. }\end{array}$ \\
\hline
\end{tabular}

\section{Visión profesional}

Para abordar la visión profesional se construyó guion de entrevista inspirada en los trabajos acerca de visión profesional docente (Van Es y Sherin 2008). Se seleccionaron dos videos de clases de ciencias naturales de cuarto básico, parte de corpus de proyectos previos del grupo de investigación. Los extractos seleccionados tratan de dos momentos típicos de clases: clase completa y trabajo en grupo de pares. En la entrevista, y a través de preguntas diseñadas cuidadosamente, se invitó a los y las participantes identificar momentos claves en el uso de argumentación en aula, explorando los fundamentos de sus decisiones y propuestas de intervención. 
Las respuestas de los y las docentes fueron codificadas de acuerdo a una rúbrica desarrollada por dos coautoras (Tabla 5) que se compone por dos partes, las cuales se usan de manera secuencial. La primera parte indaga la visión profesional a partir de la identificación de: interacciones docentes, interacciones de estudiantes, justificación de relación entre argumentación para la enseñanza y aprendizaje en ciencias, y sugerencias de intervención. Luego se sintetiza las dimensiones señaladas en una descripción única por nivel (Tabla 5). Se realizó un proceso de validación intersubjetiva por medio de reflexiones conjuntas de dos integrantes del equipo de investigación en reuniones semanales por tres meses.

TABLA 5

Rúbrica CPC de argumentación procedimental/ visión profesional - juicio global.

\begin{tabular}{|c|c|c|c|}
\hline Experto & En transición & Inicial & No experto \\
\hline $\begin{array}{c}\text { Interpreta y observa la } \\
\text { situación }\end{array}$ & $\begin{array}{c}\text { Interpreta y observa la } \\
\text { situación principalmente } \\
\text { (algunos indicadores) desde } \\
\text { untemáticamente desde } \\
\text { una perspectiva de } \\
\text { argumentación } \\
\text { centrada en la } \\
\text { discusión de ideas } \\
\text { contrarias. }\end{array}$ & $\begin{array}{c}\text { Interpreta y observa la } \\
\text { situación principalmente } \\
\text { desde una perspectiva } \\
\text { de argumentación poco } \\
\text { la discusión de ideas } \\
\text { contrarias. }\end{array}$ & $\begin{array}{c}\text { Interpreta y observa la } \\
\text { situación en parte o } \\
\text { totalmente desde una } \\
\text { perspectiva de }\end{array}$ \\
$\begin{array}{c}\text { diálogo y/o la } \\
\text { experimentación } \\
\text { científica. }\end{array}$ & $\begin{array}{c}\text { argumentación centrada } \\
\text { en la búsqueda de ideas } \\
\text { correctas o perspectiva de } \\
\text { transmisión. }\end{array}$ \\
\hline
\end{tabular}

\section{Análisis}

A partir de los análisis de datos producidos por cada instrumento, cada docente obtuvo una posición en la escala que va desde no experto a experto, en cada dimensión del CPC de la argumentación analizada. La Figura 1 muestra una síntesis de estas distintas dimensiones e instrumentos. Para poder visualizar los resultados en un continuo, se transformaron las categorías a número donde 0 corresponde a no experto, 1 a inicial, 2 a en transición y 3 a experto. Por último, y para efectos de comprensión de los resultados, se obtuvo un puntaje promedio entre las tres dimensiones de CPC de la argumentación procedimental. Si bien no se trata de variables escalares, pensamos que facilita la presentación de resultados como punto de partida. Luego, se presentan los resultados desagregados por tipo de CPC de la argumentación procedimental.

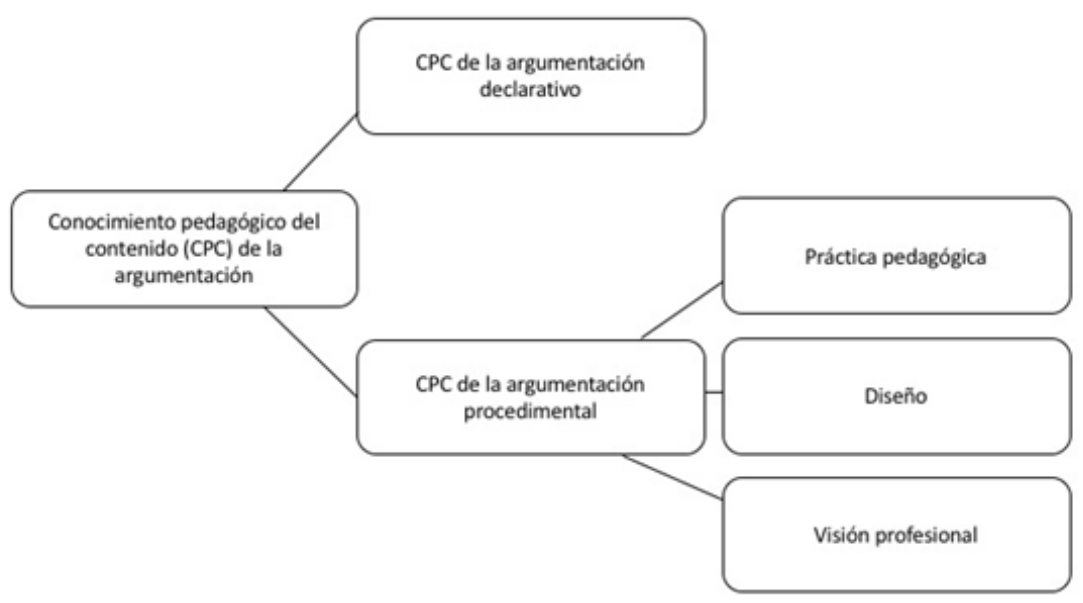

FIGURA 1

Resumen de dimensiones del CPC de la argumentación descritos. 


\section{Resultados}

Respecto al CPC declarativo, y como se puede apreciar en la Figura 2, ninguno de los docentes alcanza una puntuación que lo clasifique en el nivel experto y cuatro de diez docentes son clasificados en nivel no experto (casos 2, 3, 4 y 7). Este grupo comparte conocimiento declarativo sobre la argumentación centrado en la búsqueda de ideas correctas o de transmisión de conocimiento. Por ejemplo, ante la pregunta qué es la argumentación, encontramos:

$\ll$ Es poder defender lo que uno quiere explicar ¿Ya? Argumentar es hablar con bases, es hablar desde lo que se sabe, desde lo que aprendí y desde lo que sabe [...] es explicar algo de la forma más precisa que se puede hacer» . (Caso 2).

Un segundo grupo de docentes (casos 5, 6, 8 y 9) son clasificados en nivel inicial reconociendo principalmente la dimensión comunicativa de la argumentación en base a la experimentación científica:

$<<$ Después de haber experimentado, después de haber analizado, de haber tenido hipótesis, después de todo eso, el poder argumentar mi trabajo, mi desarrollo, me va a llevar a tener una buena calidad digamos en cuanto a mi pensamiento, a mi estructura, y a mi forma de trabajo >>. (Caso 5).

Finalmente, dos docentes son clasificados en un nivel transición donde refieren de alguna forma a la dimensión epistémica de la argumentación en su relación con el pensamiento sin que sea del todo claro cómo las distintas dimensiones de la argumentación (comunicativa, epistémica, dialéctica y pedagógica) se relacionan:

$\ll$ Decirles a los chiquillos, ponte en otro lado, o sea, no solamente con su experiencia, si no que saber ponerlo en otro punto de vista, argumentan por qué esto puede ser así, cosa de que ellos logren, ver más allá de lo evidente, que me diga ya; pero yo digo que eso es negro, y piensa que, no importa que está equivocado, no importa que esté... si, sabemos que es blanco, pero, y de alguna forma, con, con algunas ideas previas, pueda justificar».(Caso 1).

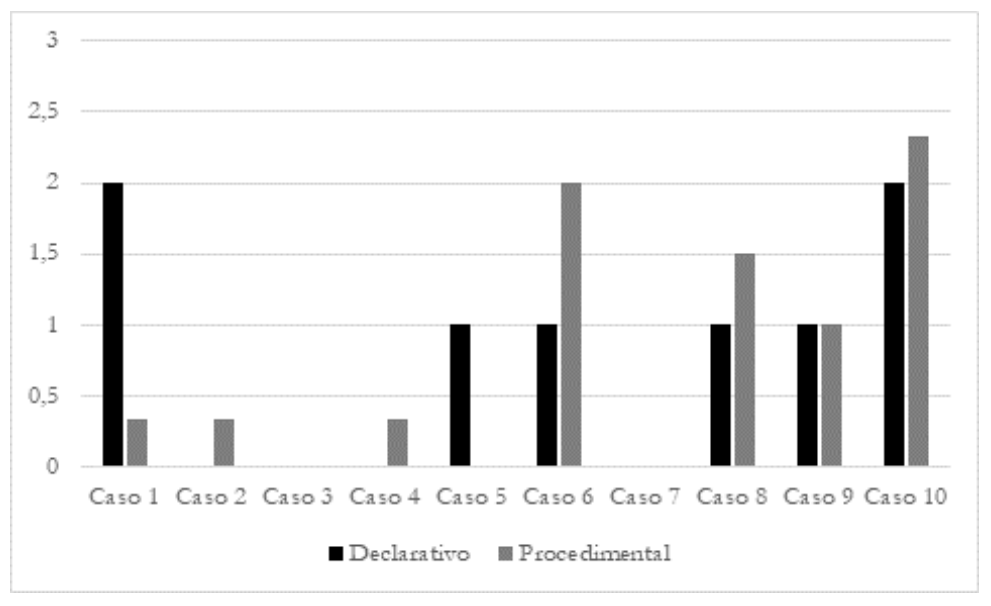

FIGURA 2

Puntaje CPC declarativo y promedio CPC procedimental por caso.

En el caso de CPC procedimental, y tal como se ve en la Figura 3, al colapsar las 3 dimensiones (visión profesional, diseño y argumentación clase ampliada) se observa que la mayor parte de los y las docentes $(6$ de 10) se ubica en un nivel no experto o muy cercano a este (casos 1, 2, 3, 4, 5 y 7 ). De los cuatro restantes, dos son clasificados en nivel inicial (casos 8 y 9) y 2 en nivel transición (casos 6 y 10). De esta manera, lo primero que se observa, es que la dimensión del CPC declarativo y procedimental no van siempre de la mano, habiendo docentes que muestran conocimiento de una dimensión y no de la otra, como el caso 2, 4 y 5.

$\mathrm{Al}$ desagregar los resultados por cada una de las dimensiones del CPC procedimental, observamos que en el caso de la visión profesional dos de ocho casos (3 y 5) son clasificados en un nivel no experto. Estos 
docentes observan e interpretan las situaciones de aula desde una perspectiva transmisiva y de verificación de los aprendizajes:

$\ll O$ sea, bueno, hay que tener claro los términos, los conceptos, [...] para poder argumentar bien, con buen fundamento $\gg$.(Caso 3).

Cuatro docentes (casos 1, 2, 4 y 10) son clasificados en el nivel inicial, interpretando las interacciones observadas principalmente desde una perspectiva comunicativa. La argumentación aparece como una forma de diálogo que complementa la experimentación científica:

«la pregunta promueve el aprendizaje, ahora, el que los chiquillos hayan participado poco, no la promueve mucho, pero la pregunta si; qué pasó con la plasticina, o qué va a pasar con la plasticina, de hecho, es un llamado a que los chiquillos investiguen» (Caso 4 ).

Finalmente, dos docentes (casos 6 y 9) son clasificados en nivel transición interpretando la situación de aula desde la relevancia de la argumentación centrada en la discusión de ideas contrarias:

«Les hubiese hecho levantar la mano a lo mejor, y decir, ya, quiénes están de acuerdo, qué pasó con algo que dijeron [...] Entonces, preguntar a lo mejor quién está de acuerdo con esa afirmación, y por qué está de acuerdo con eso. Y quienes no están de acuerdo, y los que no están de acuerdo también decirles que argumenten por qué no».(Caso 6).

Respecto al diseño de clases, siete docentes fueron clasificados en nivel no experto (casos 1, 2, 3, 4, 5, 7 y 9), lo que implica que, durante la clase, ya sea en momentos de clase completa, plenario y/o trabajo en grupo, no se percibe un diseño que promueva diferencias de opinión que se elaboren o discutan. Un docente es clasificado en nivel inicial (caso 8), un docente fue clasificado en nivel transición (caso 6) y una docente (caso 10) fue clasificada en el nivel experto. Por ejemplo, el caso 10 en una clase solicita que en grupos de pares estudiantes elaboren modelos diferentes para representar las capas de la tierra, y luego propone que escojan el mejor modelo, a partir de la discusión y fundamentación de ideas.

Respecto al uso pedagógico de la argumentación en aula, seis de diez docentes son clasificados en nivel no experto (casos 1, 2, 3, 4, 5 y 7). Esto significa que, en las clases observadas, los enunciados argumentativos corresponden a preguntas justificativas aisladas (Ejemplo: Y las placas tectónicas ¿por quéserán importantes en nuestra tierra?, Caso 5). En nivel inicial, se encuentra un docente (caso 9) en cuya clase además de preguntas docentes, aparecen argumentos de estudiantes, aunque estos no se exploran, elaboran o discuten. Por ejemplo:

$<<$ Docente (D): Salió una burbuja, ¿por qué crees que salió una burbuja?

Estudiante (E): Por el peso que tiene la arena, lo levanta >>. (Caso 9)

Dos docentes (casos 6 y 8) son clasificados en nivel transición, observándose interacciones pseudoargumentativas, como se ejemplifica a continuación:

D: Tenemos aqui un globo desinflado ¿Un globo desinflado tiene masa o no? [Situación controversial]

E 11: Sí, porque el globo desinflado e inflado pesan lo mismo. [argumento]

D: Ustedes, el globo sin aire ¿tiene masa o no tiene masa? [Situación controversial]

E8: Cuando no tiene aire no tiene masa [argumento]

D. O sea que si lo peso no pesarán nada ¿Qué dicen? ¿qué sí o que no? [Pregunta argumentativa]

E6: quesi

$D:$ ¿Por qué si? [Pregunta justificativa]

E8: Un globo desinflado tiene masa, pero muy poquita masa, porque el globo solo pesa, pero muy poquito y con masa pesa más que cuando está desinflado [argumento]

D: ya, a ver ¿qué opina la Ester? ¿Un globo desinflado tiene masa o no? [Situación controversial]

E5: No

D: No tiene... a ver ¿por qué dice que no? [Pregunta justificativa]

E5: No sé cómo explicarlo

$D:$ a ver dame un ejemplo ¿quién le puede ayudar a la compañera a explicar que un globo desinflado no tiene masa? 
E2: porque no está inflado y no tiene aire [argumento]

D: porque no está inflado, entonces si yo voy y peso ese globo ¿no pesa nada? [Pregunta contra-fáctica]

E2: es que el globo va a pesar más con aire... que el desinflado [argumento/contra-argumento a su propio argumento]

D: Ya, uno pesa más que el otro, pero ¿puedo decir que el desinflado tiene masa o no?

E: Tienen algo en común

$D:$ ¿Tienen algo en común respecto a la masa? ¿qué podemos poner en el cuaderno?

En este caso, aunque se observan argumentos y contra-argumentos, mediados por preguntas que invitan a pensar alternativas y justificarlas, no se profundiza en las ideas y preconcepciones de los y las estudiantes de manera de tensionarlas, de evaluarlas, descartarlas y transformarlas. La interacción termina cambiando de foco a cómo identificar aspectos comunes entre diferentes objetos.

Finalmente, solo una docente (caso 10) se clasifica en nivel experto observando que los argumentos y contra-argumentos son utilizados de manera que hay un foco en el logro del proceso epistémico. Por ejemplo:

D: A ver Fernanda, escuchemos a Fernanda. Fernanda ¿Cuál crees tú que es el mejor modelo? [Situación polémica]

Fernanda: El de Valeria

D: La de Valeria

Fernanda: Porque

$P: A$ ver ¿por qué? [Pregunta justificativa]

Fernanda: Hay como una, como un trozo de tierra, que ahi están las capas, entonces asi se ve la profundidad. [argumento]

$P: Y a$

A: Profe, profe, tía.

$P$ : Ya, escucharon bien lo que dijo la Fernanda ¿cierto?

A: Si

A. Profe, hay una

P: A ver, a ver, a ver, a ver, momento, a ver Paty, ¿qué dirias tú de lo dice Fernanda?

Paty: De que, si viene bien, se ve bien la profundidad, si la capa sí, pero el núcleo debería, el núcleo deberia ir más abajo, la corteza debería. [contra-argumento]

$P$ : Entonces para ti ¿cuál es el mejor modelo?

Paty: El de Tito

P: El de Tito ¿alguien está de acuerdo con la Paty? [pregunta argumentativa]

A: Si

P: Lila, ¿por qué Lila? [pregunta justificativa]

Lila: Porque aquí, o sea se ve, este está como más desordenado, porque la corteza está abajo y debe estar arriba. [argumento]

D: Ah, la corteza es la más delgada y está en la superficie terrestre.

En este caso, no solo se ofrece una situación polémica (diferentes modelos que hay que escoger) y se formulan argumentos y contra-argumentos, sino que se hace sin perder de vista el objetivo de construcción de conocimiento: que estudiantes logren reconstruir una representación coherente del orden de las capas terrestres. De hecho, la interacción no termina aquí y la docente sigue explorando las ideas de los y las estudiantes acerca de las capas de la tierra.

Respecto al CPC procedimental, es interesante observar que las diferentes dimensiones descritas no se mueven de manera sincrónica en todos los casos. Si bien hay docentes que presentan niveles parejos en todas las dimensiones (casos 3, 5 y 7 en no experto, y caso 6 en transición), en el resto de los casos el desempeño varía sin que se pueda identificar dimensiones que son consistentemente más desafiantes que otras o algunas que vayan de la mano. El caso 6 destaca por la consistencia en el CPC procedimental, donde todas las dimensiones 
se encuentran a nivel de transición, mostrando mayor avance que el CPC declarativo. Por su parte, si bien el caso 10 muestra un CPC declarativo en transición, las dimensiones del CPC procedimental varían entre inicial (visión profesional) y experto (diseño y uso pedagógico de la argumentación en aula). Este caso sugiere que el CPC declarativo no precede necesariamente la capacidad de interpretar y observar una situación de aula y que esta tampoco es condición necesaria para la práctica pedagógica.

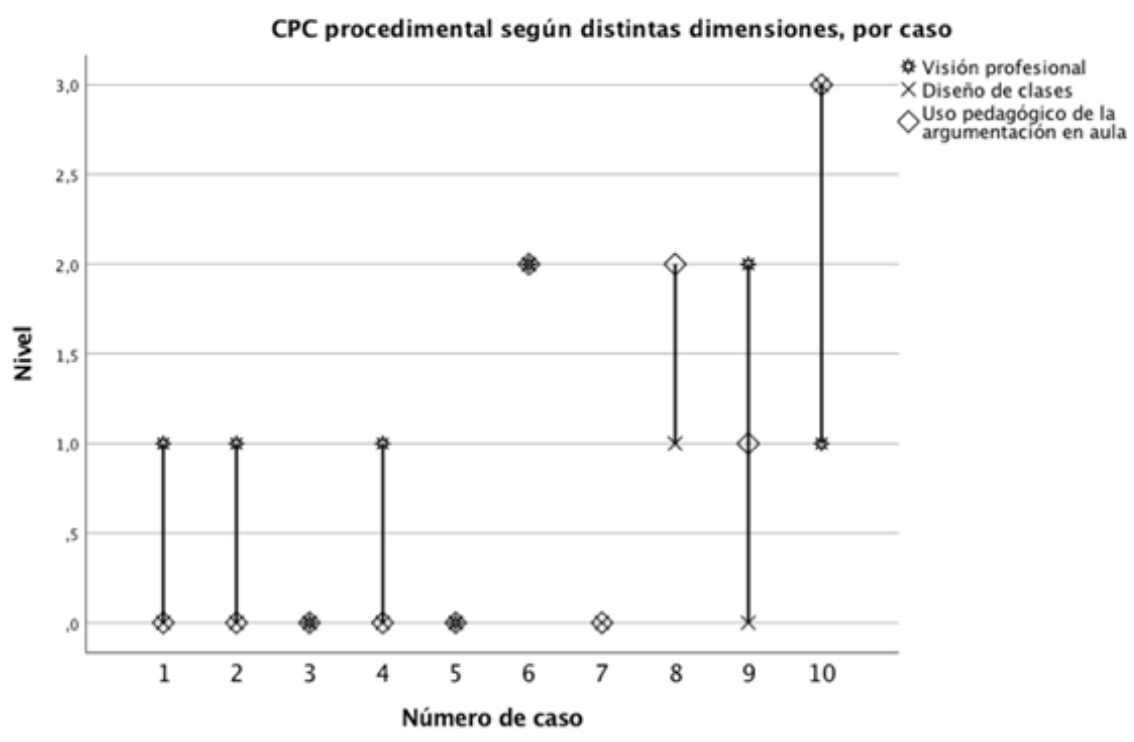

FIGURA 3

Niveles de CPC de la argumentación procedimental según distintas dimensiones por caso.

Es preciso observar que ninguna variable demográfica (sexo, edad, años de enseñanza, formación inicial o formación en indagación científica) emergen como ejes relevantes de análisis para el CPC del contenido de la argumentación de los y las docentes participantes.

\section{DiSCUSIÓN Y CONCLUSIONES}

El objetivo del estudio fue describir el nivel del CPC de argumentación de docentes que enseñan ciencias en educación primaria en Chile, de manera de contribuir a diseñar procesos de formación docente para una enseñanza de las ciencias para la ciudadanía. Aunque el estudio se trata de un estudio cualitativo acotado, sus resultados muestran distintos aspectos de interés. Por un lado, en este grupo de docentes el CPC de argumentación es un desafío para el que su formación previa (incluyendo las especializaciones en indagación científica), ni la práctica pedagógica los ha preparado del todo. Coherente con los resultados internacionales (Sadler, 2006, Wang y Buck, 2016) los y las docentes del estudio muestran en su mayoría una visión poco informada de lo que es la argumentación, su potencial de construcción de conocimiento y su desarrollo (Sampson y Blanchard 2012). Identifican los beneficios de la argumentación más con su carácter comunicativo y expresivo, que de razonamiento científico y construcción de conceptos. Esto es coherente con una visión de la enseñanza de las ciencias orientada a la transmisión del conocimiento correcto y experimentación, donde los procesos de razonamiento y persuasión colectivo no tiene rol claro, requiriendo habilidades que estudiantes no tendrían (McNeill et al. 2016). Más aún, coincidente con los resultados del estudio de Garcia Romano et al. (2016), si bien los y las docentes valoran la argumentación como forma de expresión de puntos de vista y construcción de explicaciones, no reconocen el aspecto retórico, que es parte central de la construcción de conocimiento científico. 
En términos de los conocimientos procedimentales, existe un bajo uso pedagógico de la argumentación, lo que es coherente con la evidencia disponible (González-Weil et al. 2012, Cofré et al. 2010, Larrain et al. 2014), incluso en su aspecto de construcción de explicaciones y argumentos. De hecho, no se observan reflexiones acerca de la estructura de argumentos científicos ni del rol de la evidencia en estos. Así, a diferencia de la progresión propuesta por Knight-Bardsley y McNeill (2016), este estudio observa prácticas dialógicas de argumentación en donde se discuten controversias, pero sin que se repare en el rol de evidencias. En este sentido, aunque esto se ha concebido como indicando un nivel experto, no se trataría de un nivel experto equivalente al observado en otros estudios internacionales. Además, coincidente con resultados de investigaciones de habla hispana con docentes en formación inicial (Archila, 2014; Martín-Gámez, 2020) docentes reportan dificultades en identificar con precisión estrategias pedagógicas para promover la argumentación en aula.

Ahora bien, es interesante observar que creencias más precisas e informadas respecto al uso pedagógico de la argumentación no derivan necesariamente en conocimiento procedimental (caso 1), ni un conocimiento procedimental más desarrollado requiere conocimiento declarativo avanzado como precondición necesaria (caso 6). Sin embargo, un nivel avanzado en el uso de argumentación en aula y diseño de clases puede relacionarse con un mayor nivel de conocimiento declarativo (caso 10). Esto sugiere una interesante hipótesis a explorar en futuros estudios: el conocimiento procedimental, particularmente la práctica y diseño pedagógico, podría fortalecer el conocimiento declarativo y la visión profesional. Esto implicaría revisar la idea de que el conocimiento declarativo y visión profesional precede necesariamente la práctica, y que es el único punto de partida para el desarrollo profesional.

Sin embargo, los resultados no solo levantan la visión de un déficit, sino que informan respecto a las condiciones y recursos de este grupo de docentes para posibles caminos de desarrollo del CPC de la argumentación. El estudio muestra que no hay una sola progresión de aprendizaje posible entre los distintos saberes involucrados, y que distintos saberes pueden tener presencias diferencialmente importantes en distintos docentes. Es posible que esto vaya de la mano con diferentes trayectorias y experiencias, sugiriendo lo que Clark y Hollinsworth (2002) señalan como aprendizajes que se han iniciado a partir de distintos dominios (práctica, cognición, consecuencias, entre otros). Así, este estudio puede servir para visualizar posibles trayectorias de formación a partir del conocimiento de las competencias actuales de docentes que inician procesos de desarrollo profesional en esta área, y así diseñar de manera acorde oportunidades para este.

Es claro a partir de los resultados que el CPC de la argumentación no es un solo saber, sino que se configura como un conjunto complejo de saberes diversos, que pueden tener distintos orígenes. Es posible incluso a partir de los resultados preguntarse si la misma categoría de CPC de la argumentación debe revisarse. Nuestra primera respuesta a esta pregunta es que es necesario entender esta noción como una hipótesis de trabajo, sin pretender que sea una descripción de la realidad. Como hipótesis de trabajo puede ser útil, aun cuando su descripción propone desafíos metodológicos importantes. Al respecto, reconocemos como otro aporte de este estudio la propuesta metodológica de evaluación de CPC de la argumentación. Nuestra aproximación no solo combina diversas fuentes de información como se sugiere en la literatura (Vergara y Cofré 2014), sino aporta con el uso de técnicas que resultaron especialmente interesantes, como los videos de clases ajenas para describir visión profesional. Si bien McNeill et al. (2016) usan viñetas de una manera similar, los videos permitieron rescatar con mayor claridad la dimensión dialógica, cuestión que ha sido considerada difícil de pesquisar. Por otro lado, las rúbricas de los distintos instrumentos sostienen una progresión de aprendizaje que incorpora distintas dimensiones de la argumentación (comunicativa, epistémica, dialéctica y pedagógica), incluyendo aportes de la literatura en enseñanza dialógica (Alexander 2017).

Ahora bien, el estudio tiene limitaciones. Lo primero es la muestra. Se trata de una muestra pequeña que es necesario ampliar en el futuro y, más importante aún, una muestra de dos grabaciones por clases para explorar un aspecto del CPC procedimental. Lo segundo, es de carácter metodológico. A partir del estudio sugerimos disminuir las preguntas de las entrevistas y focalizarlas en profundizar en el pensamiento de estudiantes y 
cómo promover su desarrollo, además de ampliar la reflexión respecto al currículum nacional y estrategias de evaluación de aprendizaje. Además, contar con la visión de docentes respecto a sus propias prácticas sería un aspecto que fortalecería la propuesta. Con todo, se considera que el estudio contribuye con información única que puede servir como punto de partida a futuros estudios y servir de insumo para el diseño de programas de formación docente.

En esta línea, el estudio sugiere que la formación en CPC del contenido de la argumentación no debiese basarse como punto de partida en la reconstrucción del conocimiento en su dimensión declarativa, sino que puede organizarse desde su dimensión práctica, dando oportunidades a docentes en formación de analizar con un foco en la argumentación, interacciones reales de aula tanto entre estudiantes y docentes como entre estudiantes. Más allá de promover reflexiones en general respecto a estas prácticas, la idea es promover observaciones focalizadas de manera de desarrollar visión profesional orientada a identificar con precisión movidas docentes que promueven argumentación y aprendizaje; ofrecer oportunidades para, ante determinadas intervenciones de estudiantes, anticipar lo que están pensando y responder de manera específica y precisa para promover la argumentación; identificar elementos del diseño pedagógico que facilitan y dificultan la promoción de argumentación en aula, entre otros. Aquí, sostener un foco el discurso no como un conjunto de proposiciones neutras que se repiten mecánicamente (por ejemplo la pregunta ¿por qué?) sino como posicionamientos retóricos y evaluativos (Boyd y Markarian, 2015) que para el logro de la promoción de la argumentación en y con estudiantes, requieren de ser performados en una secuencia ideacional apropiada y genuina. Por último, de esto se sigue la necesidad de fortalecer los espacios de formación respecto al diseño de clases para promover la argumentación, centrando el trabajo imaginativo y colaborativo en la transformación de los contenidos curriculares en problemas o controversias a discutir.

\section{Agradecimientos}

Este estudio ha sido financiado por la Agencia Nacional de Investigación y Desarrollo de Chile - ANID a través de programas FONDEF/ANID IX Concurso de Investigación Tecnológica FONDEF IT 20I0015 y FONDECYT proyectos1211920, 11200371 y 3190488.

\section{REFERENCIAS}

Abell, S. (2007). Research on science teacher knowledge. En: Abell, S., \& Lederman, N. (Eds.), Handbook of research on science education (pp. 1105-1149). Mahwah, NJ: Lawrence Erlbaum.

Acevedo-Díaz, J.A. y García-Carmona, A. (2016). “Algo antiguo, algo nuevo, algo prestado”. Tendencias sobre la naturaleza de la ciencia en la educación científica. Revista Eureka sobre Enseñanza y Divulgación de las Ciencias, $13(1), 3-19$.

Alozie, N. M., Moje, E. B., Krajcik, J. S. (2010). An analysis of the supports and constraints for scientific discussion in high school pro ject - based science. Science Education, 94(3), 395-427. https://doi.org/10.1002/sce. 20365

Andriessen, J., Schwarz, B. (2009). Argumentative design. In N. Muller Mirza \& A.-N. Perret-Clermont (Eds.), Argumentation and education (pp. 145-174). New York: Springer.

Asterhan, C. S., Schwarz, B. B. (2016). Argumentation for learning: Well-trodden paths and unexplored territories. Educational Psychologist, 51(2), 164-187. https://doi.org/10.1080/00461520.2016.1155458

Baxter, J. A. y Lederman, N. G. (1999). Assessment and measurement of pedagogical content knowledge. En: J. Gess-Newsome \& N. G. Lederman (Eds.), Examining pedagogical content knowledge: The construct and its implications for science education. (pp. 147-161). Dordrecht/Boston/London: Kluwer Academic Publishers.

Berry, A., P., Friedrichsen, y J., Loughran, (Eds.) (2015). Re-examining Pedagogical Content Knowledge in Science Education. Routledge. 
Boyd, M. P., \& Markarian, W. C. (2015). Dialogic Teaching and Dialogic Stance: Moving beyond Interactional Form. Research in the Teaching of English, 49(3), 272-296. http://www.jstor.org/stable/24398703

Bravo, P., Cofré, H. L. (2016). A new approach to capture and develop Biology Teachers' Pedagogical Content Knowledge through learning study: the case of human evolution. International Journal of Science Education. 38, (16): 2500-2527.

Carlson y Daehler (2019). The refined consensus model of pedagogical content knowledge in science education. En: Hume, A., R., Cooper y A., Borowski (Eds.) Repositioning Peagogical Content Knowledge in Teachers' Knowledge for Teaching Science. Springer.

Chan, K., y A., Hume (2019). Towards and consensues Model: Literature Review of How Scence Teachers' Pedagogical Content Knowledge is Investigated in Empirical Studies. En: Hume, A., R., Cooper y A., Borowski (Eds.) Repositioning Pedagogical Content Knowledge in Teachers' Knowledge for Teaching Science. Springer.

Clarke, D., Hollingsworth, H. (2002). Elaborating a model of teacher professional growth. Teaching and Teacher Education, 18(8), 947-967. https://doi.org/10.1016/S0742-051X(02)00053-7

Cofré, H., Camacho, J., Galaz, A., Jiménez, J., Santibáñez, D., Vergara, C. (2010). La educación científica en Chile: debilidades de la enseñanza y futuros desafíos de la educación de profesores de ciencia. Estudiospedagógicos, 36(2), 279-293. http://dx.doi.org/10.4067/S0718-07052010000200016

Davies, I. (2004). Science and citizenship education. International Journal of Science Education, 26(14), 1751-1763. https://doi.org/10.1080/0950069042000230785

Fortes, G., Larrain, A., Gómez, M. (2020). Design of a teacher training program for the development of pedagogical knowledge on argumentation content. Cogency, 12(2), 169-205.

Garcia, L. R. (2017). Concepciones sobre argumentación de futuros docentes de Biología. Enseñanza de las ciencias: revista de investigación y experiencias didácticas, (Extra), 2335-2342.

Gess-Newsome, J. (2015). A model of teacher professional knowledge and skill including PCK: Results of the thinking from the PCK summit. En: A. Berry, P. Friedrichsen, y J. Loughran (Eds.), Re-examining Pedagogical Content Knowledge in Science Education. (págs. 29- 45). Routledge.

González-Weil, C., Cortéz, M., Bravo, P., Ibaceta, Y., Cuevas, K., Quiñones, P., Abarca, A. (2012). La indagación científica como enfoque pedagógico: estudio sobre las prácticas innovadoras de docentes de ciencia en EM. Estudios Pedagógicos, 38(2), 85-102. http://dx.doi.org/10.4067/S0718-07052012000200006

Hemberger, L., Kuhn, D., Matos, F., Shi, Y. (2017). A dialogic path to evidence-based argumentive writing. Journal of the Learning Sciences, 26(4), 575-607. https://doi.org/10.1080/10508406.2017.1336714

Jimenez-Aleixandre, P., Erudran, S. (2008). Argumentation in science education: An overview. In S. Erduran \& M. P. Jimenez-Aleixandre (Eds.), Argumentation in science education: Perspectives from classroom-based research (pp. 47-70). Springer.

Knight - Bardsley, A., McNeill, K. L. (2016). Teachers' pedagogical design capacity for scientific argumentation. Science Education, 100(4), 645-672. https://doi.org/10.1002/sce.21222

Kolstø, S. D. (2000). Consensus projects: Teaching science for citizenship. International Journal of Science Education, 22(6), 645-664. https://doi.org/10.1080/095006900289714

Larrain, A., Freire, P., Howe, C. (2014). Science teaching and argumentation: One-sided versus dialectical argumentation in Chilean middle school science lessons. International Journal of Science Education, 26, 1017-1036. Hhttps://doi.org/10.1080/09500693.2013.832005

Leitão, S. (2000). The potential of argument in knowledge building. Human Development, 43, 332-360. https:/ doi.org/10.1159/000022697

Loughran, J.J., Mulhall, P., Berry, A. (2004). In search of pedagogical content knowledge in science: Developing ways of articulating and documenting professional practice. Journal of Research in Science Teaching, 41 (5), 370-391.

Loughran, Mulhall, P., Berry, A. (2008). Exploring pedagogical content knowledge in science teacher education. International Journal of Science Education, 30 (3), 1301-1320. 
Magnusson, S., Krajcik, J., Borko, H. (1999). Nature, sources, and development of pedagogical content knowledge for science teaching. In J. Gess-Newsome \& N. Lederman (Eds.), Examining Pedagogical Content Knowledge (pp. 95-132). The Netherlands: Science \& Technology Education Library, Kluwer Academic Publishers.

McNeill, K. L., González - Howard, M., Katsh - Singer, R., Loper, S. (2016). Pedagogical content knowledge of argumentation: Using classroom contexts to assess high - quality PCK rather than pseudoargumentation. Journal of Research in Science Teaching, 53(2), 261-290. https://doi.org/10.1002/tea.21252

McNeill, K., Knight, A. (2013). Teachers' pedagogical content knowledge of scientific argumentation: The impact of professional development on K-12 teachers. Science Education, 96(6), 936-972. http://dx.doi.org/10.1002/ sce. 21081

McNeill, K., Krajcik, J. (2008). Inquiry and scientific explanations: Helping students use evidence and reasoning. In J. Luft, R. Bell, \& J. Gess-Newsome (eds.). Science as inquiry in the secondary setting (pp. 121-134). National Science Teachers Association Press.

McNeill, K., González-Howard, M. Katsh-Singer, Loper, S. (2017). Moving Beyond Pseudoargumentation: Teachers' Enactments of an Educative Science Curriculum Focused on Argumentation. Science Education. 101(3) 426-457. https://doi.org/10.1002/sce.21274

Osborne, J. (2010). Arguing to learn in science: The role of collaborative, critical discourse. Science, 328(5977), 463-466. https://doi.org/10.1126/science.1183944

Padilla, K., Ponce de León, A., Rembado, F.M., \& Garritz, A. (2008). Undergraduate professors' pedagogical content knowledge: the case of 'amount of substance'. International Journal of Science Education, 30,10, 1389-1404.

Park, S. y Oliver, J. S. (2008). Revisiting the conceptualisation of Pedagogical Content Knowledge (PCK): PCK as a conceptual tool to understand teachers as professionals. Research in Science Education, 38(3), 261-284.

Ravanal, E., y F., López (2016). Mapa del conocimiento didáctico y modelo didáctico en profesionales del área biológica sobre el contenido de célula. Revista Eureka sobre Enseñanza y Divulgación de las Ciencias 13 (3), 725-742.

Rollnick, M. (2017). Learning about semi-conductors for teaching-the role played by content knowledge in pedagogical content knowledge (PCK) Development. Research in Science Education 47, 833-868.

Sadler, T. D. (2006). Promoting discourse and argumentation in science teacher education. Journal of Science Teacher Education, 17(4), 323-346. https://doi.org/10.1007/s10972-006-9025-4

Sampson, V., Blanchard, M. R. (2012), Science teachers and scientific argumentation: Trends in views and practice. Journal of Research in Science Teaching, 49, 1122-1148. http://dx.doi.org/10.1002/tea.21037

Sengul, O., Enderle, P. J., Schwartz, R. S. (2020). Science teachers' use of argumentation instructional model: linking PCK of argumentation, epistemological beliefs, and practice. International Journal of Science Education, 42(7), 1068-1086. https://doi.org/10.1080/09500693.2020.1748250

Shulman, L. S. (1986). Those who understand: Knowledge growth in teaching. Educational Researcher, 15(2), 4-14. https://doi.org/10.3102/0013189X015002004

Schmelzing, S., Van Driel, J. H., Jüttner, M., Brandenbusch, S., Sandmann, A. y Neuhaus, B. (2013). Development, evaluation, and validation of a paper-and-pencil test for measuring two components of biology teachers' pedagogical content knowledge concerning the "cardiovascular system". International Journal of Science and Mathematics Education, 11, 1369-1390.

Tippett, C. (2009). Argumentation: The language of science. Journal of Elementary Science Education, 21(1), 17-25.

van Driel, J. H., Berry, A., y Meirink, J. (2014). Research on science teacher knowledge. En: N. Lederman y S. Abell (Eds.), Handbook of research on science education, Volume II (págs. 848-870). New York: Routledge.

Van Es, E. A., Sherin, M. G. (2008). Mathematics teachers' "learning to notice" in the context of a video club. Teaching and Teacher Education, 24(2), 244-276. https ://doi.org/10.1016/j.tate.2006.11.005

Vergara, C., Cofré, H. (2014). Conocimiento pedagógico del contenido: ¿El paradigma perdido en la formación inicial y continua de profesores en Chile? Estudios Pedagógicos, 40, 323-338. http://dx.doi.org/10.4067/ S0718-07052014000200019 
Vergara, C., H., Cofré y D., Santibáñez (2021). Enseñanza de la Biología y conocimiento pedagógico del contenido: una introducción. En: Cofré, H., Vergara, C., y Spotorno, A. Enseñar evolución y genética para la alfabetización Cientifica. Ediciones Universitarias de Valparaíso.

Walan, S., Nilsson, P., \& Ewen, B.M. (2017). Why inquiry? Primary teachers' objectives in choosing inquiry- and context-based instructional strategies to stimulate students' science learning. Research in Science Education, 120.

Wang, J., Buck, G. A. (2016). Understanding a high school physics teacher's pedagogical content knowledge of argumentation. Journal of Science Teacher Education, 27(5), 577-604.https://doi.org/10.1007/ s10972-016-9476-1

\section{INFORMACIÓN ADICIONAL}

Para citar este artículo: Larrain, A., Gómez, M., Calderón, M., Fortes, G., Ramírez, F., Guzmán, V.y Cofré, V. (2022). Descripción del conocimiento pedagógico del contenido de la argumentación en docentes que enseñan ciencias naturales en educación pública en Chile. Revista Eureka sobre Enseñanza y Divulgación de las Ciencias 19(1), 1602.doi: 10.25267/Rev_Eureka_ensen_divulg_cienc.2022.v19.i1.1602 